http://jmscr.igmpublication.org/home/ ISSN (e)-2347-176x ISSN (p) 2455-0450 crossref DOI: https://dx.doi.org/10.18535/jmscr/v7i9.15

Original Article

\title{
Study of Extended Spectrum Beta lactamase producing Klebsiella pneumoniae from various clinical samples at tertiary care Hospital, Jaipur
}

\section{Pritam Kumar ${ }^{1}$, Anshu Shastri ${ }^{1}$, Vaishali Anandarshi ${ }^{1}$, Dr Prasanna Gupta ${ }^{{ }^{*}}$}

${ }^{1}$ Department of Microbiology, National Institute of Medical Sciences \& Research, NIMS University

Jaipur, 303121 (Rajasthan)

*Corresponding Author

Dr Prasanna Gupta

Professor \& Head, Department of Microbiology, National Institute of Medical Sciences \& Research NIMS University Jaipur, 303121 (Rajasthan), India

\begin{abstract}
Background: The present study was done to detect Extended spectrum beta lactamase producing Klebsiella pneumoniae.

Introduction: Extended spectrum of beta lactamase (ESBL) producing Klebsiella pneumoniae, frequently resistant to many of the antimicrobial agents. Antimicrobial resistance is associated with high morbidity, mortality, increased length of hospitalization and cost of health care.

Materials and Methods: A total 63 Klebsiella pneumoniae strains were isolated from various clinical specimens. Out of total 63 isolates of Klebsiella pneumoniae, total 31 ESBL Positive Klebsiella pneumoniae founded. ESBL production confirmed by double disc synergy test (DDST) and by phenotypic confirmatory disc diffusion test (PCDDT).

Results: Out of total 63 isolates of Klebsiella pneumoniae, total 31(49.20\%) ESBL Positive Klebsiella pneumoniae founded. Highest prevalence of ESBL producing Klebsiella pneumoniae was observed in case of Pus $80.0 \%$, followed by ET 58.3\%, Wound Swab \& Swab 50\% each, Sputum 45.4 \%, Blood 42.8\%, and urine 37.5\%. A total 31 ESBL Positive Klebsiella pneumoniae found in which 22 in Male and 09 in Female.

Conclusion: This study demonstrate that production of ESBL in Klebsiella pneumoniae is directly linked to its high antimicrobial resistance especially resistance towards aminoglycosides and cephalosporins. It is necessary to follow proper strategies to detect and prevent the emergence of resistance for appropriate and effective treatment of infections caused by Klebsiella pneumoniae.

Keywords: Klebsiella pneumoniae, ESBL, Sensitivity, Resistance.
\end{abstract}

\section{Introduction}

Klebisiella pneumoniae is an opportunistic pathogen that causes various illnesses such as urinary and respiratory tract infections and septicemia. They are Gram Negative, non motile, usually encapsulated, indole and ornithine decarboxylase negative they do not produce $\mathrm{H}_{2} \mathrm{~S}$, produce lysine decarboxylase and 
are generally positive in the Voges-Proskauer test. The size ranges from 0.3 to $1.0 \mathrm{~mm}$ in width and 0.6 to $6.0 \mathrm{~mm}$ in length ${ }^{1}$. Infections due to ESBL producing Klebsiella pneumoniae are of concern as third generation cephalosporins are commonly used for treatment of infections due to gram negative organisms. These infections are difficult to control as they are usually associated with resistance to aminoglycosides and cephalosporins $^{2}$.

Beta lactamases are enzymes that degrade the beta-lactam ring of the beta-lactam antibiotic group such as penicillin and cephalosporins. Extended spectrum beta -lactamase (ESBL) is an acquired class $A$ beta-lactamase that hydrolyzes and confers resistance to oxyimino second and third generation cephalosporins e.g. cefuroxime (CXM), cefotaxime (CTX), ceftazidime (CAZ), and ceftriaxone (CTR). This is one group of beta-lactamases that is, found in certain species of Gram negative bacilli $^{3,4}$.

ESBL occur mostly among lactose fermenting members of enterobacteriaceae such as Escherichia coli, Klebsiella species, and Enterobacter species and rarely in non lactose fermenters like Pseudomonas aeruginosa. ESBLs are clinically relevant and remain an important cause of treatment failure with cephalosporins 5 .

Extended spectrum of beta lactamase(ESBL) producing Klebsiella Pneumoniae were first reported from Germany in 1983 and since then a steady increase in resistance against cephalosporins.

Extended spectrum of beta lactamase (ESBL) producing Klebsiella pneumoniae, frequently resistant to many of the antimicrobial, show significant local variations. The majority of ESBLs are derived from the widespread plasmid mediated broad-spectrum beta-lactamase TEM-1 and SHV-1, found in Klebsiella pneumoniae and other pathogens ${ }^{6}$.

However, in spite of their good sensitivity to beta lactam antibiotics, there are growing concerns about increasing resistance of the organism to this same class of antibiotic because of ESBL production. In this study, we will isolate Klebsiella pneumoniae from different clinical samples with detection of ESBL production by different phenotypic methods and will study the antimicrobial susceptibility pattern of ESBL producing isolates.

\section{Materials and Methods}

The prospective observational study was carried out in Department of Microbiology at National Institute of Medical Sciences \& Research, Jaipur, Rajasthan. A total 63 Klebsiella pneumoniae strains were isolated from various clinical specimens of patients attending various outpatients and inpatients department at National Institute of Medical Sciences \& Research. Samples including urine, body fluids, pus, sputum, swab etc. were processed for isolation and identification of Klebsiella pneumoniae within the time period of July 2018 to December 2018.

All samples were inoculated on MacConkey and Blood agar, incubated at $37^{\circ} \mathrm{C}$ for $24 \mathrm{hrs}$, and colonies were processed according to standard procedures. Klebsiella pneumoniae isolates that were obtained as a confluent growth from the clinical specimens were included in the study. The organisms were identified on the basis of colony morphology, Gram staining and biochemical reactions ${ }^{7}$.

\section{Antimicrobial Susceptibility Testing}

Antimicrobial susceptibility testing was performed by modified Kirby Bauer's disc diffusion method according to CLSI guidelines 2018. An inoculums with a turbidity equivalent to that of a $0.5 \mathrm{McF}$ arland standard and Muller Hinton agar plates and commercially available antibiotics discs (Hi-Media, Mumbai) were used. Antimicrobial susceptibility testing was performed by modified Kirby Bauer's disc diffusion method according to CLSI guidelines 2018. An inoculums with a turbidity equivalent 
to that of a 0.5 McFarland standard and Muller Hinton agar plates and commercially available antibiotics discs (Hi-Media, Mumbai) were used. The antimicrobial discs were used Amikacin $(30 \mu \mathrm{g})$, Ampicillin $(10 \mu \mathrm{g})$, Cefepime $(30 \mu \mathrm{g}), \quad$ Meropenem $(10 \mu \mathrm{g}), \quad$ Ciprofloxacin $(5 \mu \mathrm{g})$, Cefotaxime $(30 \mu \mathrm{g})$, Gentamycin $(10 \mu \mathrm{g})$, Pipracillin/Tazobactam $(100 / 10 \mu g)$

Ceftazidime $(30 \mu \mathrm{g}), \quad$ Imipenem $(10 \mu \mathrm{g})$, Ceftriaxone(30 $\mu \mathrm{g})$, Polymyxin-B (300units) and Colistin $(10 \mu \mathrm{g})$. Results were measured and recorded as compared to that of the manufacturer interpretation charts according to the Clinical and Laboratory Standard Institute (CLSI) guideline ${ }^{8}$.

\section{Screening of ESBL-Producing Klebsiella pneumoniae}

According to the CLSI guidelines, isolates showing inhibition zone of $\leq 22 \mathrm{~mm}$ with Ceftazidime $(30 \mu \mathrm{g})$ and $\leq 27 \mathrm{~mm}$ with Cefotaxime $(30 \mu \mathrm{g})$ and $\leq 25 \mu \mathrm{mm}$ for Ceftriaxone were recorded were identified as potential ESBL producers and selected for confirmation of ESBL production using disc diffusion method.

\section{ESBL Confirmatory Tests \\ Double Disc Synergy Test (DDST) ${ }^{\mathbf{9}}$}

The isolated colonies were inoculated in peptone water broth at $35-37^{\circ} \mathrm{C}$ for $2-6 \mathrm{hrs}$. The turbidity adjust to 0.5 McFarland's standard and lawn culture were made on Mueller-Hinton agar using sterile swab. Amoxicillin-clavulanic acid disc $(20 / 10 \mu \mathrm{g})$ placed in the centre of plate. Both side of Amoxicillin-clavulanic disc, a disc of cefotaxime $(30 \mu \mathrm{g})$ and ceftazidime $(30 \mu \mathrm{g})$, were placed at the distance of $15 \mathrm{~mm}$ centre to centre from Amoxicillin-clavulanic acid disc. The plate were incubated at $37^{\circ} \mathrm{C}$ overnight. Enhancement of the zone of inhibition of third generation cephalosporin toward Amoxicillin-clavulanic acid confirmed the presence of ESBL.
Phenotypic confirmatory disc diffusion test (PCDDT) test for ESBL detection ${ }^{8}$ :

A disk of Ceftazidime $(30 \mu \mathrm{g})$ alone and a disk of Ceftazidime + Clavulanic acid $(30 / 10 \mu \mathrm{g})$ discs were placed at least $30 \mathrm{~mm}$ apart, center to center. A difference of $\geq 5 \mathrm{~mm}$ between the zone diameters of Ceftazidime and its Ceftazidime/ Clavulanic acid discs is taken to be phenotypic confirmation of ESBL production.

\section{Results}

During the study period, 63 Klebsiella pneumoniae were isolated from various clinical specimens of patients of all ages and both sexes at National Institute of Medical Sciences \& Research. Out of total 63 isolates of Klebsiella pneumoniae, $42(66.66 \%)$ isolates from male while $21(33.33 \%)$ from female patients. Out of total 63 isolates of Klebsiella pneumoniae, $03(4.76 \%)$ isolated from age 0-10yrs , 11-20yrs $9(14.28 \%), \quad 21-30 \mathrm{yrs} 11(17.46 \%), \quad 31-40 \mathrm{yrs}$ 07(11.11\%), 41-50 yrs 10 (15.87\%), 51-60 yrs $10(15.87 \%)$ and above 60 yrs $13(20.63 \%)$.

Maximum sensitivity was shown with Polymyxin-B and Colistin 100\% each, Meropenem 87.30\%, Imipenem $66.66 \%$, Amikacin, Gentamycin, and Pipracillin/ Tazobactam 57.14\% each, Ceftazidime $47.61 \%$, Ciprofloxacin 38.09\%, Ampicillin 36.5\%, Cefotaxime $31.74 \%$. minimum sensitivity shown by Ceftriaxone $26.98 \%$.

Out of total 63 isolates of Klebsiella pneumoniae, total 31(49.20\%) ESBL Positive Klebsiella pneumoniae founded and total 32(50.79\%) Non ESBL Klebsiella pneumoniae founded. Highest prevalence of ESBL producing Klebsiella pneumoniae was observed in case of Pus $80.0 \%$, followed by ET $58.3 \%$, Wound Swab \& Swab 50\% each, Sputum 45.4 $\%$, Blood $42.8 \%$, and urine $37.5 \%$. A total 31 ESBL Positive Klebsiella pneumoniae found in which 22 in Male and 09 in Female. 
Graph 1: Age wise distribution of Klebsiella pneumoniae.

\section{Age wise distribution of isolates}

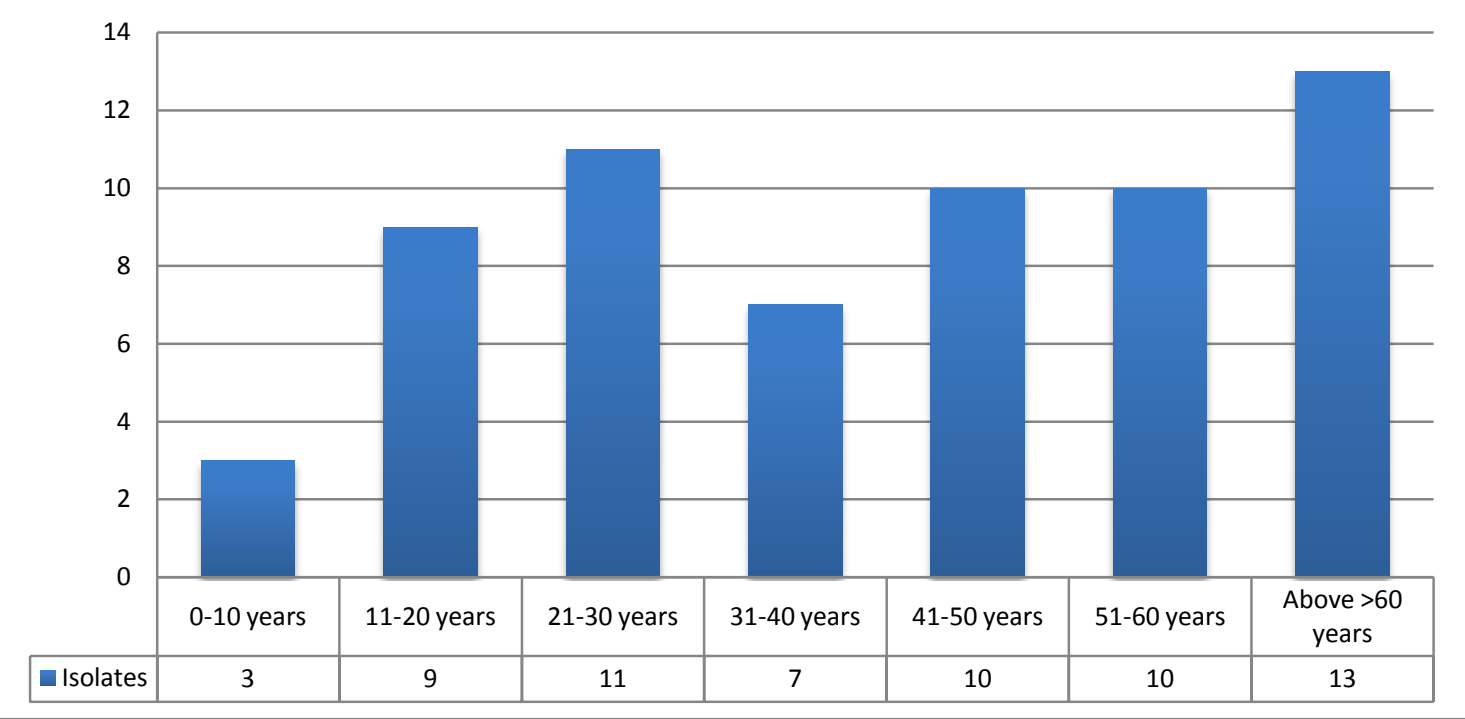

Graph 2: Antimicrobial susceptibility pattern of Klebsiella pneumoniae.

\section{Antimicrobial Susceptibility Pattern}

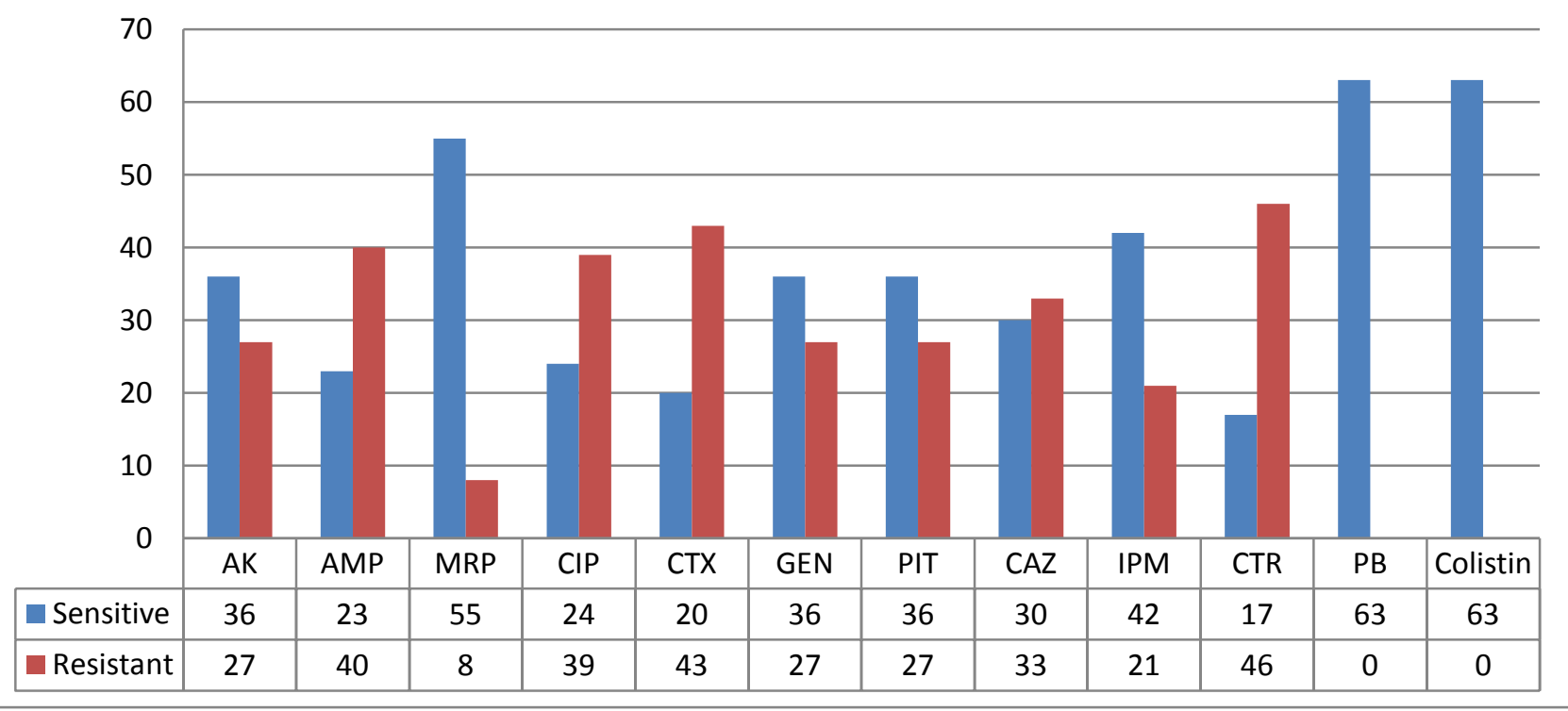

Table: 1: Antibiotic sensitivity pattern of Klebsiella pneumoniae

\begin{tabular}{|l|c|c|c|c|c|c|}
\hline S. No. & Antibiotics & Disc potency & Sensitive & Percentage \% & Resistance & Percentage \% \\
\hline 1. & Amikacin & $30 \mu \mathrm{g}$ & 36 & $57.14 \%$ & 27 & $42.85 \%$ \\
\hline 2. & Ampicillin & $10 \mu \mathrm{g}$ & 23 & $36.50 \%$ & 40 & $63.49 \%$ \\
\hline 3. & Meropenem & $10 \mu \mathrm{g}$ & 55 & $87.30 \%$ & 08 & $12.69 \%$ \\
\hline 4. & Ciprofloxacin & $5 \mu \mathrm{g}$ & 24 & $38.09 \%$ & 39 & $61.90 \%$ \\
\hline 5. & Cefotaxime & $30 \mu \mathrm{g}$ & 20 & $31.74 \%$ & 43 & $68.25 \%$ \\
\hline 6. & Gentamycin & $10 \mu \mathrm{g}$ & 36 & $57.14 \%$ & 27 & $42.85 \%$ \\
\hline 7. & Pipracillin/Tazobactam & $100 / 10 \mu \mathrm{g}$ & 36 & $57.14 \%$ & 27 & $42.85 \%$ \\
\hline 08. & Ceftazidime & $30 \mu \mathrm{g}$ & 30 & $47.61 \%$ & 33 & $52.38 \%$ \\
\hline 09. & Imipenem & $10 \mu \mathrm{g}$ & 42 & $66.66 \%$ & 21 & $33.33 \%$ \\
\hline 10. & Ceftriaxone & $30 \mu \mathrm{g}$ & 17 & $26.98 \%$ & 46 & $73.01 \%$ \\
\hline 11. & Polymyxin-B & $300 \mathrm{units}$ & 63 & $100 \%$ & 00 & $00 \%$ \\
\hline 12. & Colistin & $10 \mu \mathrm{g}$ & 63 & $100 \%$ & 00 & $00 \%$ \\
\hline
\end{tabular}


Graph 3 : Total No. of ESBLs Positive Klebsiella pneumoniae

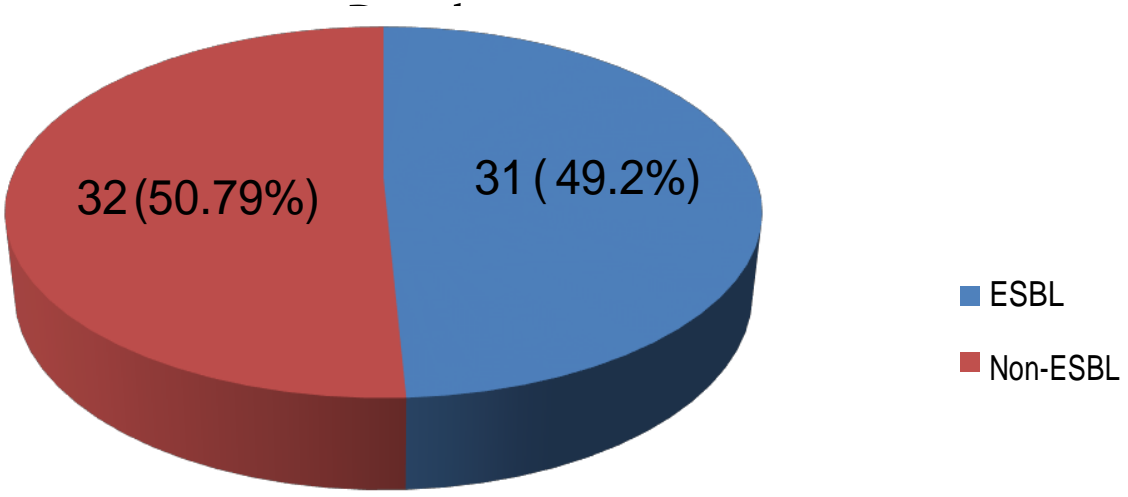

Table 2: Distribution of ESBLs Positive Klebsiella pneumoniae from various clinical sample

\begin{tabular}{|l|l|l|l|l|l|l|}
\hline S. No. & Specimen & $\begin{array}{l}\text { No. of Isolates K. } \\
\text { pneumoniae }\end{array}$ & ESBL & Percentage \% & Non-ESBL & Percentage \% \\
\hline 1. & Urine & 16 & 06 & $37.5 \%$ & 10 & $62.5 \%$ \\
\hline 2. & Blood & 07 & 03 & $42.85 \%$ & 04 & $57.14 \%$ \\
\hline 3. & ET & 12 & 07 & $58.33 \%$ & 05 & $41.66 \%$ \\
\hline 4. & Sputum & 11 & 05 & $45.45 \%$ & 06 & $54.54 \%$ \\
\hline 5. & Ear Swab & 02 & 00 & $00.0 \%$ & 02 & $100 \%$ \\
\hline 6. & Pus & 10 & 08 & $80.00 \%$ & 02 & $20 \%$ \\
\hline 7. & Wound Swab & 02 & 01 & $50.00 \%$ & 01 & $50.00 \%$ \\
\hline 8. & Swab & 02 & 01 & $50.00 \%$ & 01 & $50.00 \%$ \\
\hline 9. & Semen & 01 & 00 & $00.0 \%$ & 01 & $100 \%$ \\
\hline & Total & $\mathbf{6 3}$ & $\mathbf{3 1}$ & $\mathbf{4 9 . 2 0 \%}$ & $\mathbf{3 2}$ & $\mathbf{5 0 . 7 9 \%}$ \\
\hline
\end{tabular}

Graph 4: Distribution of ESBL producing Klebsiella pneumoniae in Male and Female

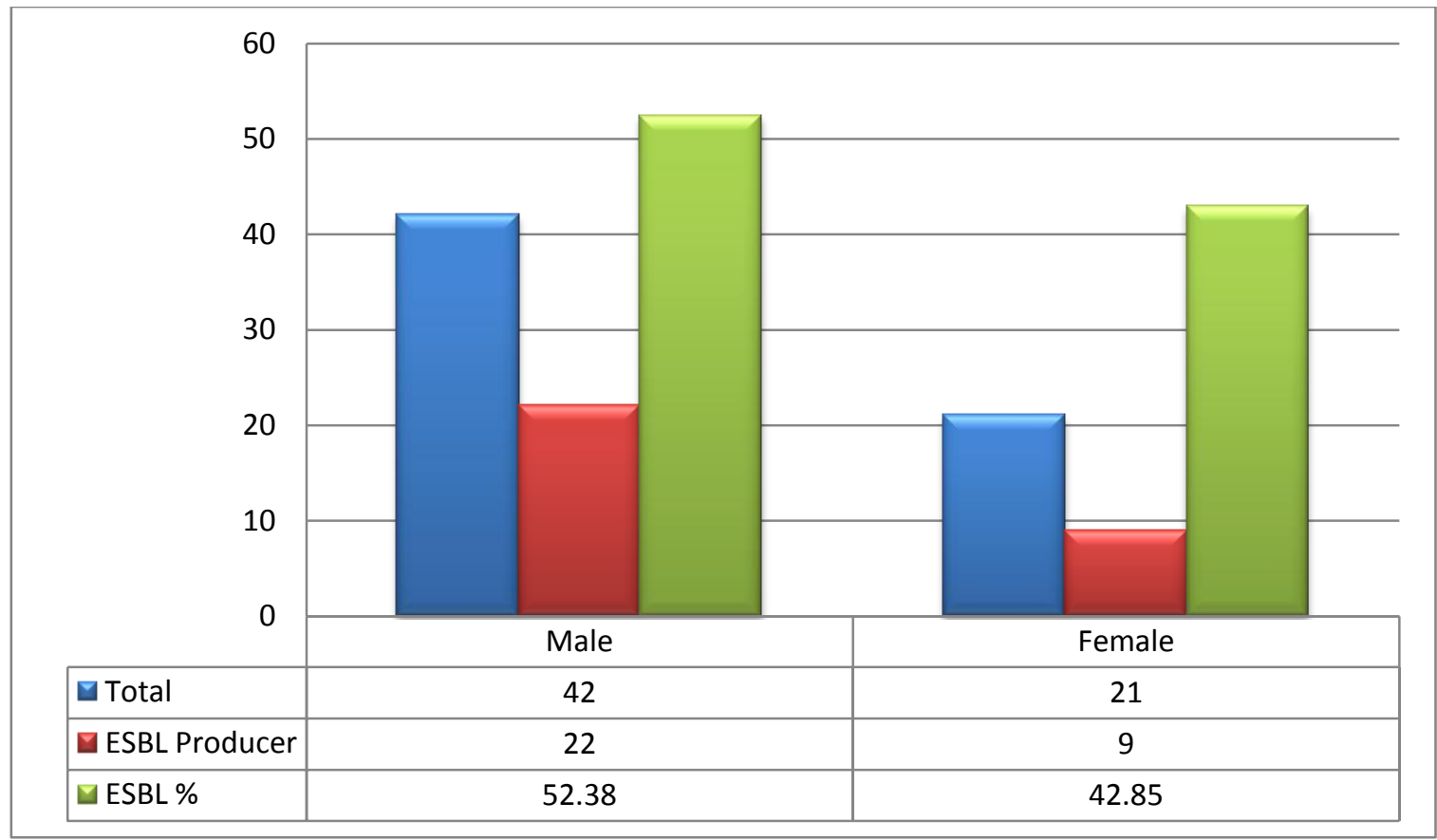




\section{Discussion}

The present study was conducted in the Department of microbiology, National Institute of Medical Sciences \& Research, NIMS University, Jaipur Rajasthan, from July 2018 to Dec 2018. During the study period, total 63 positive strains of Klebsiella pneumoniae were isolated from various clinical specimens at NIMS hospital. Klebsiella pneumoniae was common in male patients i.e. $66.66 \%$ as compare to female patients i.e. $33.33 \%$. Similar observation of male preponderance than female was seen by Sourav Chakraborty et al $2016^{10}$ i.e. $57 \%$ in male and $43 \%$ in female and Priyadarshini M. Deodurg, et al $2014^{11}$,i.e. $56.66 \%$ in male and $44.16 \%$ in female. On the other hand according to Akila.K et al $2016^{12}$, Klebsiella pneumoniae observed in $38.76 \%$ in male and $61.24 \%$ in female. In the present study, out of 63 Klebsiella pneumoniae strains isolated from different age group, the maximum no. of isolates from above 60 years old patients, i.e. $20.63 \%$ which correlate with Shristi Raut et al $2015^{13}$ i.e. $23.5 \%$. In the present study, out of 63 Klebsiella pneumoniae isolated from various clinical samples, maximum no. of isolates from urine $25.39 \%$ followed by ET $19.04 \%$, Sputum $17.46 \%$, Pus $15.87 \%$, and blood $11.11 \%$, which correlate with Akila. K et al $2016^{12}$, i.e. Urine $52.15 \%$, Sputum 29.67\%, Pus $17.22 \%$ and Blood $0.96 \%$. In the present study out of 63 Klebsiella pneumoniae, ESBL producing Klebsiella pneumoniae is $49.20 \%$ whereas according to Khalid Abdalla Ali Abdel Rahim et al $2014^{15}$, No. of Klebsiella pneumoniae producing ESBL was $53.84 \%$. In other study done by Sourav Chakraborty et al $2016^{15}$, Klebsiella pneumoniae producing ESBL $53 \%$. According to our study among 63 Klebsiella pneumoniae, ESBL producing isolates from pus were $80 \%$, Endotracheal Tube $58.33 \%$, Wound swab50.0\%, Sputum $45.45 \%$, Blood $42.85 \%$, and Urine $37.5 \%$. As compare to the study of Akila. K et al $2016^{12}$, i.e. ESBL producing strains isolated from Urine $40.0 \%$, Sputum
$38.75 \%$, Pus $21.25 \%$. A study made by Faari $\mathrm{BU}$ et al, $2015^{16}$, isolation rate of ESBL in Klebsiella pneumonaie from Swabs was $55.7 \%$ followed by Blood $17.1 \%$, Urine $14.28 \%$, sputum $12.85 \%$. The prevalence of ESBLs $(49.20 \%)$ in the present study was parallel from different parts of the country (14-74\%). According to this study, Out of 63 isolates of Klebsiella pneumoniae, maximum sensitivity were against Polymyxin -B \& Colistin 100\% each followed by Meropenem 87.3\%, Imipenem $66.66 \%$, Amikacin, Gentamycin and Piperacillin/ tazobactam $57.14 \%$ each, Ceftazidime $47.61 \%$, Ciprofloxacin 38.09\%, Ampicillin 36.5\%, Cefotaxime $31.74 \%$, and minimum sensitivity shown by Ceftrixone $26.98 \%$. In comparison to Khalid Abdalla Ali Abdel Rahim et al 2014 ${ }^{14}$, approximately Imipenem $84.61 \%$, Amikacin 76.92\%, Gentamycin 53.84\%, Pipracillin/Tazobactam $76.92 \%$, Ciprofloxacin $30.10 \%$, Cefepime and Cefotaxime $23.07 \%$ each. It has been proved that the prevalence of the ESBLs among the clinical isolates varies from country to country and institution within the same country. This might be due to judicious usage of Extended Spectrum Cephalosporins and adopting appropriate infection control measures in hospital.

\section{Conclusion}

This study demonstrate that production of ESBL in Klebsiella pneumoniae is directly linked to its high antimicrobial resistance especially resistance towards aminoglycosides and cephalosporins. This study shows that ESBL producing strains were resistant to routinely prescribed Antimicrobial agents which is an alarming sign for healthcare workers. Present study will guide the physicians to choose drug which can be used in the infection caused by the Klebsiella pneumoniae in our centre. Therefore it is necessary to follow proper strategies to detect and prevent the emergence of resistance for appropriate and effective treatment of infections caused by Klebsiella pneumoniae. 


\section{References}

1. Cantón R, Novais A, Valverde A, Machado E, Peixe L, Baquero F, et al. Prevalence and spread of extendedspectrum betalactamase producing Enterobacteriaceae in Europe. Clin Microbiol Infect 2008;14 (Suppl 1):14453.

2. Ananthakrishan AN, Kanungo R, Kumar A, Badrinath S. Detection of extended spectrum beta-lactamase producers among surgical wound infections and burn patients in JIPMER. Indian $\mathrm{J}$ Med Microbiol 2000;18:160-165

3. Aibinu IE, Ohaegbulam VC, Adenipekun EA, Ogunsola FT, Odugbemi TO, Mee BJ. Extendedspectrum beta-lactamase enzymes in clinical isolates of Enterobacter species from Lagos, Nigeria. J Clin Microbiol 2003;41:2197-200.

4. Bradford PA. Extended-spectrum betalactamases in the 21st century: Characterization, epidemiology, and detection of this important resistance threat. Clinical Microbiology Rev 2001;14:933-51.

5. Cantón R, Novais A, Valverde A, Machado E, Peixe L, Baquero F, et al. Prevalence and spread of extendedspectrum beta-lactamaseproducing Enterobacteriaceae in Europe. Clin Microbiol Infect 2008;14 (Suppl 1):14453.

6. Haque S. Associate professor, Department of medicine, J.N. Medical College, AMU, ALIGARH(2011). Extended spectrum beta-lactamase (ESBL) mediated resistance in urinary tract infections changing profile at a teaching hospital of north India, International Journal of Current Biological and Medical Sciences ,2011; 1(3):103-107.

7. Collee, J.G., Miles, R.S. and Watt, B.
(1996) Tests for the Identification of Bacteria. In: Collee, J.G., Marmion, B.P., Fraser, A.G. and Simmons, A., Eds., Mackie \& McCartney Practical Medical Microbiology, 14th Edition, Churchill Livingstone, New York, 131151.

8. Clinical and laboratory standards institute (CLSI) 2018. Performance standards for antimicrobial Susceptibility testing, $28^{\text {th }}$ Ed Wayne, USA.

9. V. Jarlier, M. H. Nicolas, G. Fournier, and A. Philippon, "Extended broad-spectrum beta-lactamases conferring transferable resistance to newer beta-lactam agents in Enterobacteriaceae: hospital prevalence and susceptibility patterns," Reviews of Infectious Diseases, vol. 10, no. 4, pp. 867-878, 1988.

10. Sourav Chakraborty, Kaniz Mohsina, Palash Kumar Sarker, Md Zahangir Alam, M Ismail Abdul Karim, S M Abu Sayem, Prevalence, antibiotic susceptibility profiles and ESBL production in Klebsiella pneumoniae and Klebsiella oxytoca among hospitalized patients, Periodicum Biologorum UDC 57:61 Vol. 118, No 1, 53-58, 2016.

11. Priyadarshini M. Deodurg, Rajive Kumar Sureka, Ravi D. Mala, Prevalence and antibiogram of extended spectrum $\beta$ lactamase producing Klebsiella pneumoniae in a tertiary care hospital, Journal of Scientific and Innovative Research 2014;3(2):155-159.

12. Akila. K, Nithyalakshmi.J, Mohanakrishnan. K, Sumathi.G Prevalence of ESBL Producing Klebsiella Species and Their in-Vitro Antimicrobial Susceptibility Pattern in A Tertiary Care Hospital. IOSR Journal of Dental and Medical Sciences (IOSRJDMS) 2016 Volume 15, Issue 11 Ver. 
VIII, PP 05-10.

13. Shristi Raut, Shishir Gokhale, Bipin Adhikari, Prevalence of Extended Spectrum Beta- Lactamases among Escherichia coli and Klebsiella spp isolates in Manipal Teaching Hospital, Pokhara, Nepal, Journal of Microbiology and Infectious Diseases/2015;5(2):69-75.

14. Khalid Abdalla Ali Abdel Rahim , Ahmed Mohamed Ali Mohamed, Prevalence of Extended Spectrum $\beta$ lactamase-Producing Klebsiella pneumoniae in Clinical Isolates, Jundishapur J Microbiol. 2014 November; 7(11): e17114.

15. Sourav Chakraborty, Kaniz Mohsina, Palash Kumar Sarker, Md Zahangir Alam, M Ismail Abdul Karim, S M Abu Sayem, Prevalence, antibiotic susceptibility profiles and ESBL production in Klebsiella pneumoniae and Klebsiella oxytoca among hospitalized patients, Periodicum Biologorum UDC 57:61 Vol. 118, No 1, 53-58, 2016.

16. Faari B U, Akanbi A A, Fa deyi A, Wahab K W, Nwabuisi C. Prevalence of extended spectrum beta-lactamase-producing Klebsiella species at the University of Ilorin Teaching Hospital.J Med Investig Pract 2015;10:20-23. 